\title{
Postcards from the EDge: 5-year outcomes of a randomised controlled trial for hospital- treated self-poisoning
}

\author{
Gregory L. Carter, Kerrie Clover, Ian M. Whyte, Andrew H. Dawson and Catherine D'Este
}

\section{Background}

Repetition of hospital-treated self-poisoning and admission to psychiatric hospital are both common in individuals who self-poison.

\section{Aims \\ To evaluate efficacy of postcard intervention after 5 years. \\ Method \\ A randomised controlled trial of individuals who have self- poisoned: postcard intervention (eight in 12 months) plus treatment as usual $v$. treatment as usual. Our primary outcomes were self-poisoning admissions and psychiatric admissions (proportions and event rates).}

\section{Results}

There was no difference between groups for any repeatepisode self-poisoning admission (intervention group: $24.9 \%$,
95\% Cl 20.6-29.5; control group: 27.2\%, 95\% Cl 22.8-31.8) but there was a significant reduction in event rates (incidence risk ratio (IRR) $=0.54,95 \% \mathrm{Cl} 0.37-0.81$ ), saving 306 bed days. There was no difference for any psychiatric admission (intervention group: $38.1 \%$, 95\% Cl 33.1-43.2; control group: $35.5 \%, 95 \% \mathrm{Cl} 30.8-40.5)$ but there was a significant reduction in event rates (IRR $=0.66,95 \% \mathrm{Cl}$ 0.47-0.91), saving 2565 bed days.

\section{Conclusions}

A postcard intervention halved self-poisoning events and reduced psychiatric admissions by a third after 5 years. Substantial savings occurred in general hospital and psychiatric hospital bed days

\section{Declaration of interest}

None.
Hospital-treated self-poisoning is common in Australia ${ }^{1,2}$ and hospital-treated self-harm is common in the UK., ${ }^{3,4}$ Self-poisoning is the most common variant of self-harm and most patients make initial contact with hospital through the emergency department. ${ }^{5}$ Repetition of self-harm is common; with, within 1-4 years of an index episode, a median rate of $21 \%$ and after more than 4 years a median rate of $23 \%{ }^{6}$ Self-harm has a strong association with subsequent suicide as well as significant resource implications for the health system. ${ }^{6}$ Only three interventions in randomised controlled trials have reported reductions in the proportion of participants with repetition of self-harm beyond the duration of the intervention: 5-month follow-up after four sessions of interpersonal therapy; ${ }^{7} 18$-month ${ }^{8}$ and 5 -year follow-up ${ }^{9}$ for a day hospital programme using mentalisation techniques; and 12-month follow-up ${ }^{10}$ for dialectical behaviour therapy. One study has demonstrated a reduction in the event rate of selfpoisoning after 12 months of follow-up. ${ }^{11}$ Only two trials have reported reductions in admissions to psychiatric hospitals beyond the duration of the intervention; hospital-based mentalisation and dialectical behaviour therapy. ${ }^{10}$ One study has demonstrated the cost-benefits for hospital-based mentalisation after 36-month follow-up, with no cost differences between the groups during pre-treatment or treatment and costs of partial hospital treatment offset by less psychiatric in-patient care and reduced emergency room treatment. ${ }^{12}$

In this study we aimed to test the efficacy of a postcard intervention in hospital-treated patients who had self-poisoned over 5 years (4 years' follow-up after cessation of the intervention). There were two primary outcomes: general hospital admissions for repetition of self-poisoning and psychiatric admissions for any reason. For each primary outcome we examined differences in proportions for any admission and event rates for the number of admissions per person. Secondary binary end-points were all-cause mortality and suicide.

\section{Method}

The study methods for Postcards from the EDge have been previously reported in detail. ${ }^{13}$ The Hunter Area Toxicology Service (HATS) is a regional toxicology unit at the Calvary Mater Newcastle, New South Wales, Australia, serving a primary referral population of 385000 adults and a tertiary referral population of a further 170000 at the time the study commenced. All poisoning presentations to emergency departments in the greater Newcastle region are either admitted to HATS or notified to HATS and entered prospectively into a clinical database. ${ }^{14,15}$ The HATS model of service delivery means that (virtually) all individuals who have self-poisoned in this catchment area are brought to the Calvary Mater Newcastle for treatment and all of them are formally admitted under the care of HATS. Tertiary referrals from outside this immediate catchment area (the Hunter Valley) are also accepted for admission. The psychiatry department sees all patients who have self-poisoned for assessment, diagnosis and to determine discharge destination and follow-up. Details of the model of service for these patients have been previously described. ${ }^{14}$ The Hunter Research Ethics Committee (HAREC) approved this study, including the randomised-consent design (Project Number: 9710153.15).

\section{Study population}

All individuals who self-poisoned and were aged 16 years or over who presented to HATS during the recruitment period (April 
1998 to December 2001) were potentially eligible. Inclusion criteria were: capable of informed consent, not considered to pose a threat to an interviewer, not of 'no fixed address' and sufficient English to complete a structured interview.

\section{Study variables}

The dependent variables after 5 years were as follows for the primary end-points: (a) the proportion of individuals who had one or more admissions to the general hospital for self-poisoning and the number of self-poisoning admissions per individual, determined from the HATS database; (b) the proportion of individuals who had one or more admissions to any public psychiatric hospital (serving the greater Newcastle area and the Hunter Valley) and the number of admissions per individual, extracted from the Hunter New England Area Health Services (HNEAHS) Clinical Information System. The secondary endpoints were the number of all-cause deaths and suicide deaths, determined by data linkage with the Australian Institute of Health and Welfare (AIHW) mortality database.

The characteristics of the participants were obtained from the standardised clinical assessment of patients who self-poison ${ }^{16}$ at index self-poisoning admission and extracted from the HATS database. The categorical variables were: gender, marital status (married or de facto $v$. never married, separated, divorced or widowed), employment (full time or part time $v$. unemployed, pensioner, student or other), intensive care unit (ICU) admission, time of admission (09.00-17.00 h weekdays $v$. out of hours) and discharge destination (psychiatric hospital $v$. all others). The continuous variables were: age, length of stay in hours, median number of previous self-poisoning admissions to HATS and number of psychiatric diagnoses from clinical assessment.

\section{Design}

A randomised-consent (single-consent version) design was used. ${ }^{17,18}$ This design is a variation on the standard randomised controlled experimental design, where participants are randomised to control or intervention before consent is sought. In the single-consent version, written informed consent to receive the intervention (eight non-obligatory postcards) is sought only from participants randomised to the intervention. The participants were masked to the study outcomes. The outcomes were assessed by an intention-to-treat analysis based on randomisation status.

\section{Randomisation}

Randomisation was by database (HanDBase version 2.0, DDH Software, Wellington, Florida, USA) on a personal digital assistant (Palm III, Palm Inc., Sunnyvale, California, USA), which was populated with a pre- generated randomisation schedule (in blocks of ten) and carried by the duty toxicologist. To avoid recruiting patients more than once, identification information was searched in this database before enrolment. To maintain masking to allocation status during recruitment, randomisation was not revealed until after all information was entered and eligibility determined. Randomisation status was then revealed in order to obtain patient consent. To monitor any potential alterations (interference) a duplicate record was kept in a hidden field of the database and a copy held on a separate computer for later verification of correct randomisation status. All other clinical and research staff were kept masked to allocation.

\section{Intervention}

The intervention was based on the study by Jerome Motto that demonstrated reduced death by suicide in a population in a psychiatric hospital. ${ }^{19,20}$ The new intervention was a series of eight 'postcards' sent in a sealed envelope in months 1, 2, 3, 4, 6, 8, 10 and 12 after discharge. All intervention and control participants received treatment as usual (TAU) (Fig. 1).

\section{Sample sizes}

During study planning several sample sizes were calculated based on different estimates of possible effects for the 12-month outcomes. ${ }^{21}$ A difference in proportions (5\% significance level, $80 \%$ power) of 15 to $10 \%$ required 1364,20 to $10 \%$ required 392 and 30 to $20 \%$ required 293 participants. Based on clinical experience and previous research it was anticipated that between 15 and $30 \%$ of control group participants would repeat selfpoisoning within 12 months, with an average of two episodes, meaning overall self-poisoning rates of 30 to $60 \%$. A sample of 400 per group would allow detection of absolute differences between groups of 10 to $15 \%$ ( $5 \%$ significance level, $80 \%$ power) and 12 to $17 \%$ (5\% significance level, $90 \%$ power), yielding relative risks of 0.67 to 0.75 and 0.60 to 0.72 for $(80 \%$ and $90 \%$ power respectively). This sample size would be adequate to detect differences in the proportion of participants who had any selfpoisoning of $7-9 \%$ ( $80 \%$ power) and $8-10 \%$ (90\% power and $5 \%$ significance level), which we considered would represent a clinically significant reduction.

\section{Statistical analyses}

Data were analysed using computerised statistical packages, PASW Statistics 18 and Intercooled Stata Version 10 on Windows. Graphs were prepared using Microsoft Excel 2010 on Windows.

The difference in proportions with any self-poisoning readmission or any psychiatric admission was tested with chisquared. For the number of self-poisoning readmissions (events) per individual and number of psychiatric admissions per individual, negative binomial regressions were undertaken to compare the risk of events per individual in the postcard group relative to the control group and is reported as incidence risk ratios (IRR with 95\% CIs). Two subgroup analyses for selfpoisoning and psychiatric admission events using negative binomial regression models were undertaken: for the treatment effect for gender since a post hoc analysis of 12-month outcomes suggested a gender effect; ${ }^{13}$ and for baseline history of previous self-poisoning $v$. no previous self-poisoning, since this might be associated with differential repetition rates for self-poisoning ${ }^{22}$ and subsequent psychiatric hospital admission. ${ }^{23}$

For the binary outcomes, relative risk reduction (RRR) and number needed to treat (NNT with 95\% CIs) were calculated using the online calculator at http://statpages.org/nntcalculator. html.

\section{Intent to treat}

A total of 922 patients were assessed for eligibility, with 150 (16\%) ineligible, leaving 772 participants (394 in the control group, 378 in the intervention group - Fig. 1). Among the intervention group, 76 refused the intervention and 1 missed the intervention as planned; 32 did not receive the full intervention, with some of their postcards being returned 'not known at this address - return to sender'. Twenty controls received the intervention due to clerical errors and were retained in the control group for analyses. All analyses were as intention to treat, based on randomisation; 378 intervention and 394 control participants were included for analyses. 


\section{Results}

\section{Characteristics of the study participants at baseline}

The characteristics of the participants by randomisation group at baseline can be seen in Table 1. Since HATS has primary and tertiary referral populations, we also examined the two groups at baseline for place of residence and found no differences; for the immediate catchment area for primary referrals only (control group $n=311,81 \%$; intervention group $n=301,81 \%$ ), for the intermediate area with a mixture of primary and tertiary referrals (control group $n=65,17 \%$; intervention group $n=64,17 \%$ ), for the remote catchment area with only tertiary referrals (control group $n=9,2 \%$; intervention group $n=8,2 \%$ ) and missing place of origin data $(n=14)$. We had missing place of residence data for controls $(n=9)$ and cases $(n=5)$. The poisons used in the index self-poisoning episode were classified as: pharmaceuticals only, 473 (61\%); pharmaceuticals plus alcohol, 217 (28\%); opioid or amphetamine, 20 (3\%); carbon monoxide, 17 (2\%); herbicide or rodenticide, $11(1 \%)$; insulin, $7(1 \%)$; any self-harm with self-poisoning, 23 (3\%); and unknown poison, 4 (1\%).

\section{Proportion of participants with any repeat episode of general hospital-treated self-poisoning}

After 5 years, 94 participants $(24.9 \%$, 95\% CI 20.6 to 29.5) in the intervention group had one or more repeat episodes of hospital-treated self-poisoning compared with 107 participants (27.2\%, 95\% CI 22.8 to 31.8 ) in the control group (difference between groups $2 \%, 95 \%$ CI -4.0 to 8.0$)$ - a non-significant difference $\left(\chi^{2}=0.41\right.$, d.f. $\left.=1, P=0.520\right)$. The RRR was $0.08(95 \%$ CI -0.16 to 0.28 ) and the NNT was 43.68 (95\% CI -26 to 12 ).

The 76 participants who refused the intervention as allocated by randomisation were included in the results above. For these 76 participants in the intervention group, 56 (male $n=19$, female $n=37$ ) had no repetition; 13 (male $n=5$, female $n=8$ ) had 1 repetition, and for multiple repetitions 1 male accounted for 3 episodes and 6 females accounted for 30 episodes.

\section{Number of repeat episodes of admission to the general hospital for self-poisoning}

There were 484 self-poisoning readmissions in the control group and 252 in the intervention group, a difference of 232 readmissions (Fig. 2). The number of episodes was significantly reduced in the intervention group compared with controls, $\mathrm{IRR}=0.54$ (95\% CI $0.37-0.81)$. Separate subgroup analyses by gender showed that the treatment was effective for females (IRR $=0.54,95 \%$ CI 0.34-0.88), but not males (IRR 1.11, 95\% CI 0.55-2.24).

Subgroup analyses by baseline history of prior self-poisoning showed that the treatment was effective for the prior self-poisoning subgroup $($ IRR $=0.39,95 \%$ CI $0.18-0.81)$ but not significantly

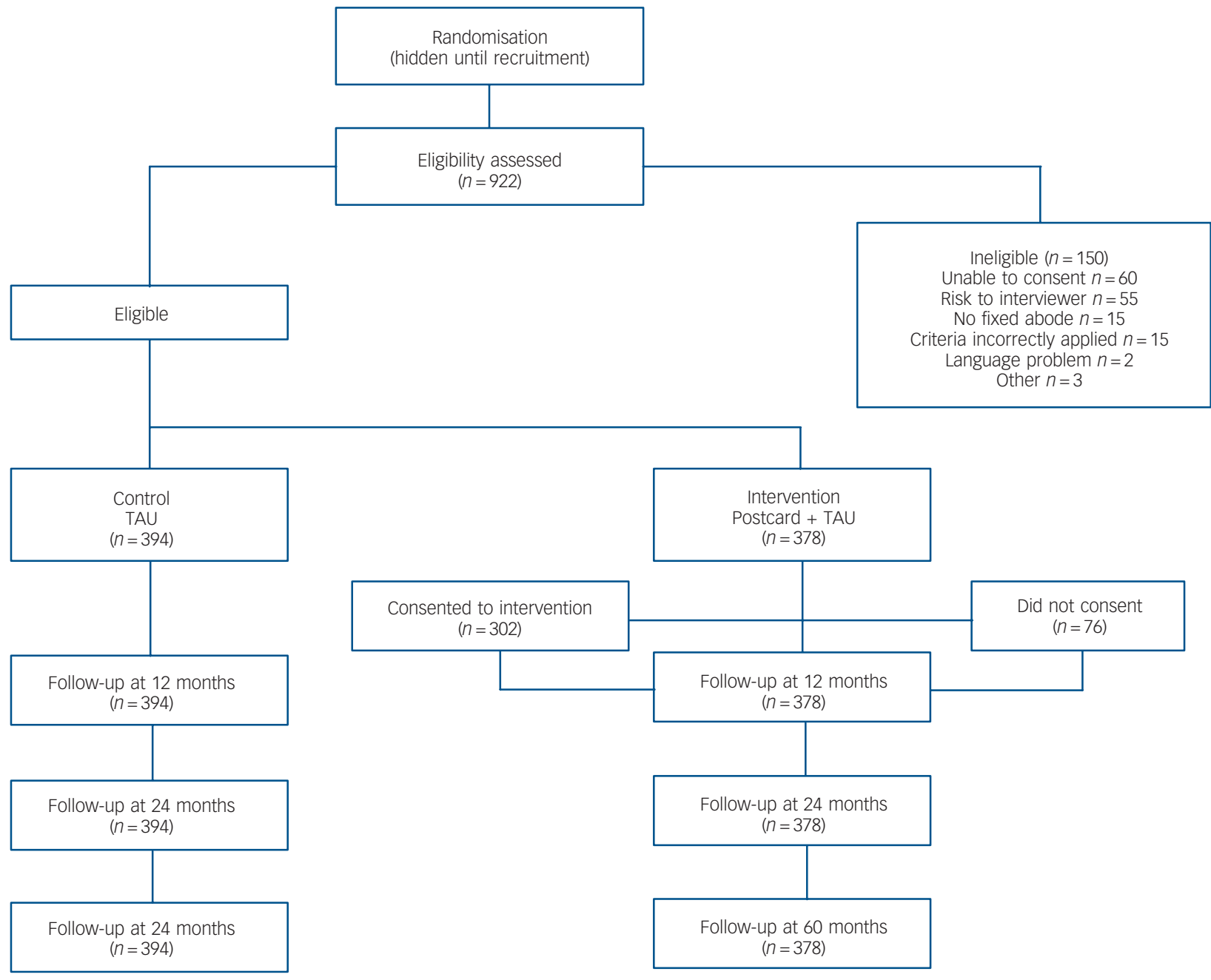

Fig. 1 Consort diagram for randomised design with 5-year follow-up. 


\begin{tabular}{|c|c|c|c|c|}
\hline Characteristic & $\begin{array}{c}\text { Total } \\
(n=772)\end{array}$ & $\begin{array}{l}\text { Control } \\
(n=394)\end{array}$ & $\begin{array}{l}\text { Intervention } \\
\qquad(n=378)\end{array}$ & $\begin{array}{c}\text { Missing data } \\
n\end{array}$ \\
\hline \multicolumn{5}{|l|}{ Categorical variables, $n$ \% } \\
\hline Female & $524(68)$ & $291(74)$ & $233(62)$ & 1 \\
\hline Married/de facto & $256(35)$ & $118(31)$ & $138(38)$ & 31 \\
\hline Employed & $162(26)$ & $88(27)$ & $74(26)$ & 153 \\
\hline Admitted to intensive care & $113(15)$ & $60(15)$ & $53(14)$ & 0 \\
\hline Admitted outside office hours & $581(75)$ & $296(75)$ & $285(75)$ & 0 \\
\hline Discharged to psychiatric hospital & $208(27)$ & $106(27)$ & $102(27)$ & 2 \\
\hline Previous self-poisoning admission & 129 (17) & $66(17)$ & $63(17)$ & 0 \\
\hline \multicolumn{5}{|l|}{ Continuous variables, median (Q1-3) ${ }^{\mathrm{a}}$} \\
\hline Age, years & $33(24-44)$ & $34(23-45)$ & $33(24-42)$ & 0 \\
\hline Length of stay, hours & $18(12-30)$ & $18(13-31)$ & $17(12-29)$ & 0 \\
\hline Number of prior self-poisoning admissions & $0(0)$ & $0(0)$ & $0(0)$ & 0 \\
\hline Number of psychiatric diagnoses & $2(1-2)$ & $2(1-3)$ & $2(1-3)$ & 0 \\
\hline
\end{tabular}

different for the no prior self-poisoning subgroup (IRR 0.76, 95\% CI 0.49-1.18) (Table 2).

The total duration of self-poisoning readmissions in the general hospital was 641 bed days for the control group and 335 bed days for the intervention group, a difference of 306 days.

\section{Proportion of participants with any psychiatric hospital admission}

In the intervention group $144 / 378$ (38.1\%, 95\% CI 33.1 to 43.2$)$ had one or more psychiatric hospital admissions compared with $140 / 394(35.5 \%, 95 \%$ CI 30.8 to 40.5$)$ in the control group (absolute difference between groups $-3.0 \%, 95 \%$ CI -9.0 to 4.0), a non-significant difference $\left(\chi^{2}=440\right.$, d.f. $\left.=1, P=0.507\right)$. The RRR was -0.07 ( $95 \% \mathrm{CI}-0.29$ to 0.11 ) and the NNT was -39.03 (95\% CI -11 to 24$)$.

\section{Number of episodes of admission to a psychiatric hospital}

There were 710 admissions in the control group and 447 in the intervention group, a difference of 263 admissions (Fig. 3). The number of psychiatric admissions was significantly lower in the intervention group compared with the control group $(\mathrm{IRR}=0.66,95 \%$ CI $0.47-0.91)$. Separate subgroup analyses by gender showed no statistical difference for females $(\mathrm{IRR}=0.70$, 95\% CI $0.46-1.07$ ) or males (IRR $=0.67,95 \%$ CI $0.40-1.13$ ), although both subgroups showed similar levels of reduced IRR. Subgroup analysis by baseline history of prior self-poisoning showed no statistical difference for the no prior self-poisoning subgroup (IRR $=0.76,95 \%$ CI $0.54-1.09$ ) or for the prior selfpoisoning subgroup (IRR $=0.53,95 \%$ CI $0.26-1.07$ ), with both showing somewhat similar levels of reduced IRR (see Table 3 ).

The total duration of admissions to a psychiatric hospital was 6008 bed days in the control group and 3443 bed days in the intervention group, a difference of 2565 days.

\section{All-cause mortality 5 years post-randomisation}

There were 44 (5.7\%) all-cause deaths, with 22/378 (5.8\%, 95\% CI 3.7 to 8.7 ) in the intervention group and $22 / 394$ (5.6\%, 95\% CI 3.5 to 8.3 ) in the control group (absolute difference between groups $-0.2 \%, 95 \% \mathrm{CI}-3.0$ to 3.0 ), a non-significant difference

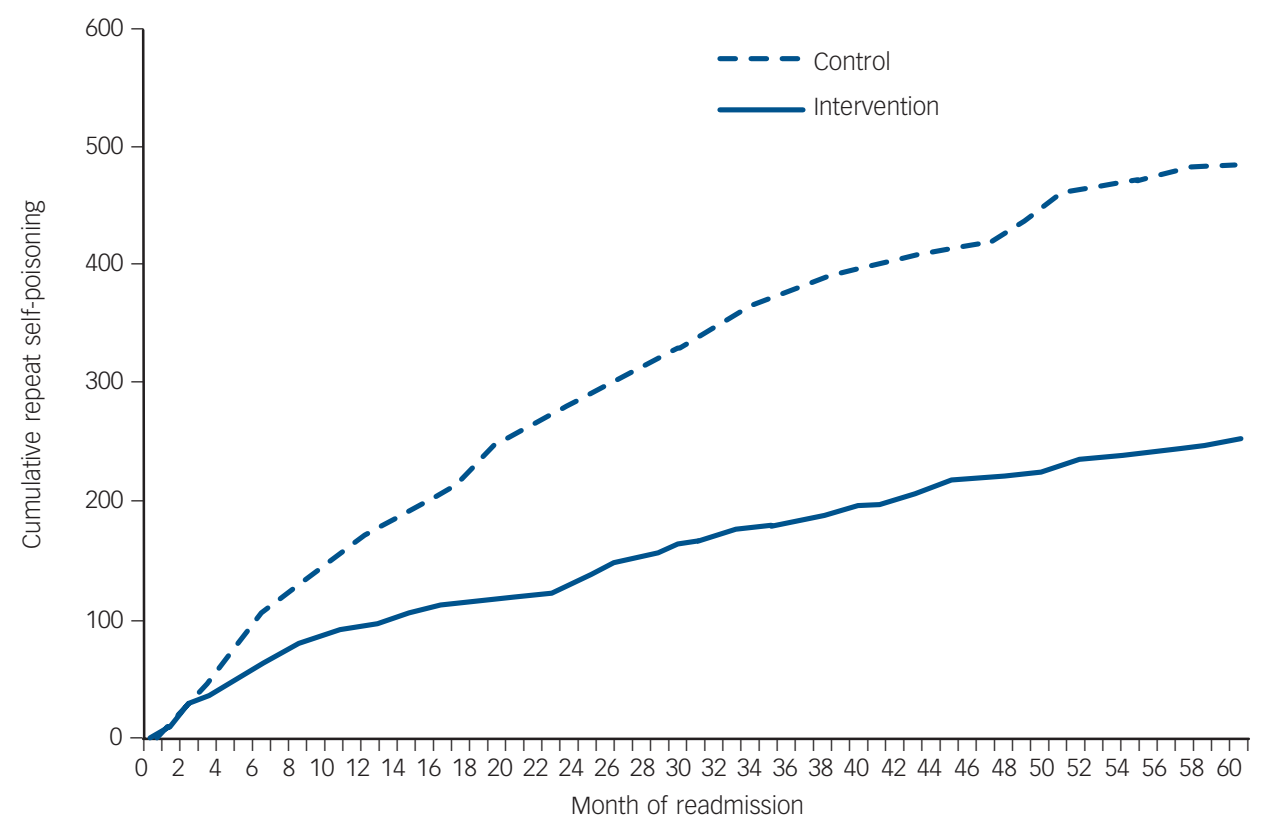

Fig. 2 Cumulative 5-year readmission for hospital-treated self-poisoning. 


\begin{tabular}{|c|c|c|c|}
\hline & Incidence risk ratio $(95 \% \mathrm{Cl})$ & $Z$-value & $P$ \\
\hline \multicolumn{4}{|c|}{ Group (unadjusted) } \\
\hline Control & 1.00 & & \\
\hline Intervention & $0.54(0.37-0.81)$ & -3.00 & $<0.01$ \\
\hline \multicolumn{4}{|c|}{ Subgroup analyses ${ }^{\mathrm{a}}$} \\
\hline \multicolumn{4}{|c|}{ Men $(n=247)$} \\
\hline Control & 1.00 & & \\
\hline Intervention & $1.11(0.55-2.24)$ & 0.30 & 0.77 \\
\hline \multicolumn{4}{|l|}{ Women $(n=524)$} \\
\hline Control & 1.00 & & \\
\hline Intervention & $0.55(0.34-0.88)$ & -2.49 & 0.01 \\
\hline \multicolumn{4}{|c|}{ No prior self-poisoning $(n=643)$} \\
\hline Control & 1.00 & & \\
\hline Intervention & $0.76(0.49-1.18)$ & -1.20 & 0.23 \\
\hline \multicolumn{4}{|c|}{ Prior self-poisoning $(n=129)$} \\
\hline Control & 1.00 & & \\
\hline Intervention & $0.39(0.18-0.81)$ & -2.54 & 0.01 \\
\hline
\end{tabular}

$\left(\chi^{2}=0.00\right.$, d.f. $\left.=1, P=1.00\right)$. The RRR was $-0.042(95 \%$ CI -0.85 to 0.41$)$ and the NNT was -423.10 (95\% CI -28 to 32$)$.

\section{Suicide deaths 5 years post-randomisation}

There were 11 (1.4\%) suicides in the study population, with 5/378 $(1.3 \%, 95 \%$ CI 0.4 to 3.1$)$ in the intervention group and $6 / 394$ $(1.5 \%, 95 \%$ CI 0.6 to 3.3$)$ in the control group (absolute difference between the groups $0.2 \%, 95 \% \mathrm{CI}-1.0$ to 2.0 ), a non-significant difference $\left(\chi^{2}=0.00\right.$, d.f. $\left.=1, P=1.00\right)$. The RRR was $0.13(95 \% \mathrm{CI}$ -0.82 to 0.73 ) and the NNT was 499.77 (95\% CI -58 to 47 ).

\section{Discussion}

\section{Strengths and weaknesses of the study}

This study had several strengths. A randomised-consent (Zelen) design was used, which was suited to this study and this clinical population. The quality of the randomisation was strong with randomisation undertaken using a handheld personal computer device in combination with another computer that would detect any errors of allocation status and any interference with randomisation. All participants were masked to study end-points. The principal outcomes were tracked by the HATS database for general hospital-treated episodes of self-poisoning and by the HNEAHS statistical service for psychiatric hospital admissions, allowing complete follow-up for the primary outcomes; with secondary outcomes derived from data linkage with national mortality data, which should also provide complete detection of end-points at follow-up. Using these databases to determine end-points should also eliminate response bias (no participant report required) and ensured masking to allocation for the raters. We used an intention-to-treat analysis based on randomisation status. We believe that there are few threats to internal validity in this study.

There were some limitations to consider for this study and some caution needs to be used when interpreting the results. The toxicologists responsible for participant recruitment and the

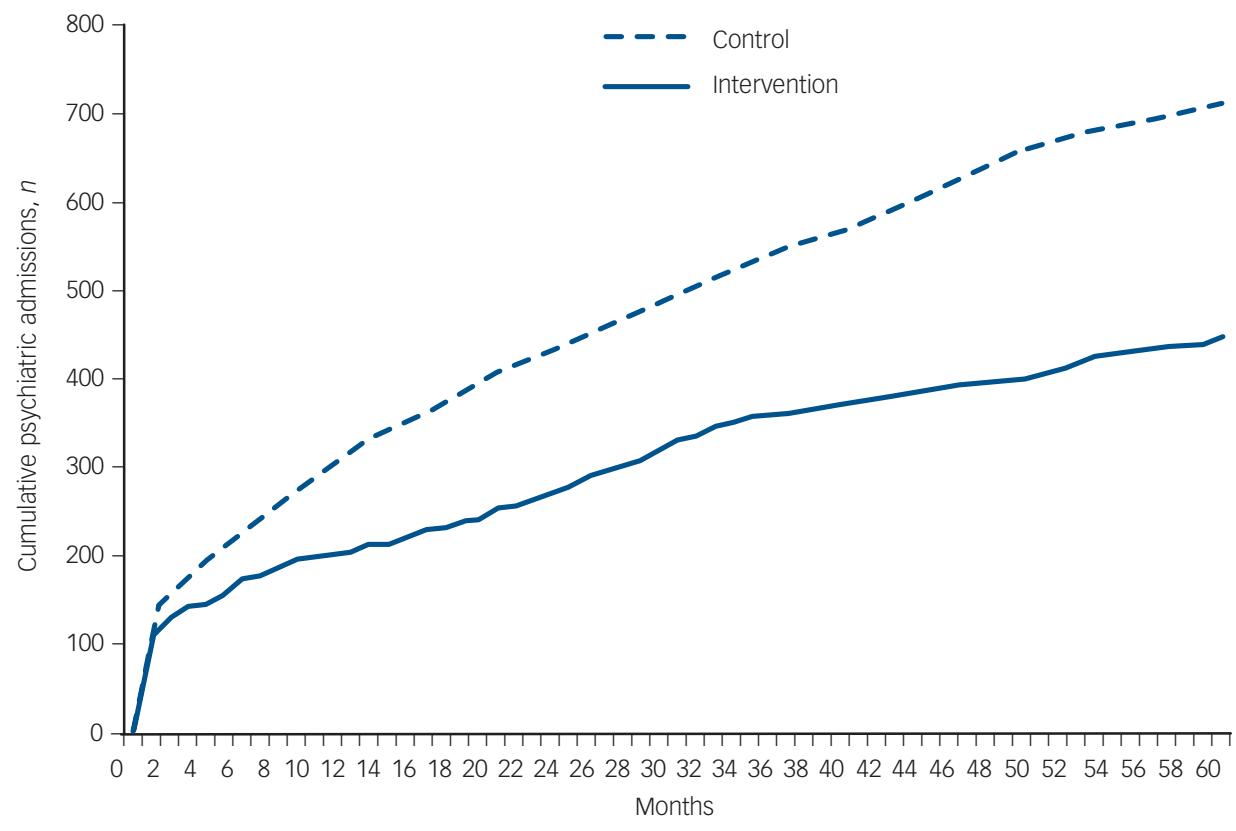

Fig. 3 Cumulative 5-year psychiatric hospital admissions. 


\begin{tabular}{|c|c|c|c|}
\hline & Incidence risk ratio $(95 \% \mathrm{Cl})$ & Z-value & $P$ \\
\hline \multicolumn{4}{|c|}{ Group (unadjusted) } \\
\hline Control & 1.00 & & \\
\hline Intervention & $0.66(0.47-0.91)$ & -2.51 & 0.01 \\
\hline \multicolumn{4}{|c|}{ Subgroup analyses ${ }^{a}$} \\
\hline \multicolumn{4}{|c|}{ Men $(n=247)$} \\
\hline Control & 1.00 & & \\
\hline Intervention & $0.67(0.40-1.13)$ & -1.49 & 0.14 \\
\hline \multicolumn{4}{|l|}{ Women $(n=524)$} \\
\hline Control & 1.00 & & \\
\hline Intervention & $0.70(0.46-1.07)$ & -1.67 & 0.10 \\
\hline \multicolumn{4}{|c|}{ No prior self-poisoning $(n=643)$} \\
\hline Control & 1.00 & & \\
\hline Intervention & $0.76(0.54-1.09)$ & -1.50 & 0.13 \\
\hline \multicolumn{4}{|c|}{ Prior self-poisoning $(n=129)$} \\
\hline Control & 1.00 & & \\
\hline Intervention & $0.53(0.26-1.07)$ & -1.76 & 0.08 \\
\hline
\end{tabular}

administrative assistant responsible for managing the mailing database and postcards were unmasked for allocation status after the point of recruitment. The HATS and HNEAHS databases would not account for admissions to hospitals outside of the respective catchment areas, meaning that the reported end-points would be underestimates, however we would expect no differential effects in a randomised design. There may also have been a conservative estimate of efficacy of treatment effect because 20 controls were inadvertently exposed to the intervention; and 76 participants in the intervention group did not consent to receive the intervention and a further 32 participants were not exposed to the complete intervention.

Around $25 \%$ of participants in the study had a repeat episode of hospital-treated self-poisoning and they showed a highly skewed distribution of number of repeat episodes. Around 37\% of the participants in the study had a subsequent psychiatric hospital admission with a similar skewed distribution of number of admissions. This means that these end-points were restricted to a small proportion of the overall study group. Caution should always be used in interpreting subgroup analyses because of (unplanned) reduced sample sizes and judgement also needs to be exercised regarding the biological or psychological plausibility of such analyses. The subgroup analyses based on gender and previous hospital-treated self-poisoning at baseline were post hoc and relatively underpowered, so the possibility of a failure to detect a significant difference needs to be considered. The decision to include subgroup analyses based on gender and previous selfpoisoning was based on the known differential rates of repetition of hospital-treated self-poisoning and psychiatric hospital admissions in our clinical population. ${ }^{21-23}$

The external validity is not known. In particular, the characteristics of the HATS referral population, the model of clinical service for self-poisoning, ${ }^{14}$ the rates of repetition of hospital-treated self-poisoning, the rates of psychiatric hospital admissions after index self-poisoning event and the ready availability of aftercare from mental health services, might not be generalised to other settings. The results of the intervention might not be generalised to patients with other forms of self-harm.

\section{Implications of the study}

This simple postcard intervention, sustained over 12 months, has been previously shown to have nearly halved the number of selfpoisoning readmissions within 12 months $($ IRR $=0.55,95 \%$ CI
$0.35-0.87) ;^{13}$ a benefit maintained after 24 months $(\mathrm{IRR}=0.49$, 95\% CI $0.33-0.73) .{ }^{11}$ For the 5-year follow-up, this benefit was maintained $($ IRR $=0.54,95 \%$ CI $0.37-0.81$ ), with a saving of 306 bed days from 232 fewer admissions in the general hospital. The 5-year rate of repetition in the control group was 27.2\% (95\% CI 22.8-31.8), which was similar to the pooled median rate of $23 \%(\mathrm{IQR}=11-32)$ for repeated self-harm after more than 4 years' follow-up, reported in a systematic review. ${ }^{6}$

We have reported for the first time, a reduction of about a third in the number of psychiatric hospital admissions (IRR $=0.66$, 95\% CI $0.47-0.91$ ), saving 2565 bed days from 263 fewer admissions.

The difference in total repeat admissions to the general hospital for self-poisoning came mainly from women with 3 or more repeat admissions, which accounted for a difference of 226 admissions (345 in the control group and 119 in the intervention group). The difference in total repeat admissions to the psychiatric hospital also came mainly from women with 3 or more repeat admissions, which accounted for a difference of 248 admissions (482 in the control group and 234 in the intervention group) (Table 4).

We considered the reduction in admissions and bed days in the general hospital and the psychiatric hospital to be clinically significant. This also represents a considerable saving in opportunity cost in availability of hospital beds and decrease in emergency department and psychiatric hospital workload. We did not make a formal evaluation of cost-effectiveness. The simplicity of the intervention and the low cost means that it could be delivered from hospitals that do not have extensive resources for patients who self-poison.

\section{Subgroup analyses}

We selected two subgroups for analysis because of known differences in repetition rates of hospital-treated self-poisoning and psychiatric hospital admissions for any reason (higher repetition in females and those with a prior self-poisoning), rather than any specific expectation of differential treatment effect based on theoretical grounds for which subgroups might be responsive. There was a different pattern of results for the two primary outcomes.

The magnitude of effect for self-poisoning events (nearly 50\% reduction) was similar to the effect for females in the 12 -month ${ }^{13}$ and 24 -month studies ${ }^{11}$ and for suicide attempt after 12 months in a replication study, ${ }^{24}$ whereas the IRR was close to unity for 


\begin{tabular}{|c|c|c|c|c|c|c|c|c|}
\hline \multirow[b]{3}{*}{ Number of repetitions } & \multicolumn{4}{|c|}{ Control group ${ }^{\mathrm{a}}(n=394)$} & \multicolumn{4}{|c|}{ Intervention group $(n=378)$} \\
\hline & \multicolumn{2}{|c|}{ Male $(n=102)$} & \multicolumn{2}{|c|}{ Female $(n=291)$} & \multicolumn{2}{|c|}{ Male $(n=145)$} & \multicolumn{2}{|c|}{ Female $(n=233)$} \\
\hline & $\begin{array}{l}\text { Participants } \\
\quad n(\%)\end{array}$ & $\begin{array}{c}\text { Admissions } \\
n\end{array}$ & $\begin{array}{l}\text { Participants } \\
\quad n(\%)\end{array}$ & $\begin{array}{c}\text { Admissions } \\
n\end{array}$ & $\begin{array}{l}\text { Participants } \\
n(\%)\end{array}$ & $\begin{array}{c}\text { Admissions } \\
n\end{array}$ & $\begin{array}{l}\text { Participants } \\
\quad n(\%)\end{array}$ & $\begin{array}{c}\text { Admissions } \\
n\end{array}$ \\
\hline \multicolumn{9}{|c|}{$\begin{array}{l}\text { Admission to general hospital } \\
\text { for self-poisoning }\end{array}$} \\
\hline 0 & $80(78)$ & 0 & $207(71)$ & 0 & $116(80)$ & 0 & $168(72)$ & 0 \\
\hline 1 & $13(13)$ & 13 & $41(14)$ & 41 & $16(11)$ & 16 & $33(14)$ & 33 \\
\hline 2 & $5(5)$ & 10 & $18(6)$ & 36 & $5(3)$ & 10 & $16(7)$ & 32 \\
\hline 3 or more & $4(4)$ & 20 & $25(9)$ & 345 & $8(6)$ & 42 & $16(7)$ & 119 \\
\hline \multicolumn{9}{|l|}{$\begin{array}{l}\text { Admission to psychiatric } \\
\text { hospital for any reason }\end{array}$} \\
\hline 0 & $65(64)$ & 0 & $189(65)$ & 0 & $88(61)$ & 0 & $146(63)$ & 0 \\
\hline 1 & $13(13)$ & 13 & $46(16)$ & 46 & $32(22)$ & 32 & $42(18)$ & 42 \\
\hline 2 & $8(8)$ & 16 & $13(4)$ & 26 & $12(8)$ & 24 & $17(7)$ & 34 \\
\hline 3 or more & $16(16)$ & 114 & $43(15)$ & 482 & $13(9)$ & 81 & $28(12)$ & 234 \\
\hline
\end{tabular}

males, suggesting a beneficial effect for females but not males. However, the picture is not so clear for those with prior selfpoisoning at baseline. Although there was a significant effect for those with prior self-poisoning, there was a non-significant effect in the same direction for those without prior self-poisoning. Patients with prior self-poisoning have substantially higher repetition rates and so the significant finding here might be because of the power to detect a difference or to a treatment effect in favour of those with prior self-poisoning, although we have not tested for effect modification by prior self-poisoning.

For the psychiatric admission outcome, there was no statistically significant effect for gender subgroups (due to lack of power), although the magnitude of effect (around 30\% reduction) was very similar for males and females, suggesting equal efficacy for this outcome for the two subgroups. There was a non-significant reduction in psychiatric admission events for prior self-poisoning or no prior self-poisoning, which may be because of insufficient power or because there was no treatment effect for this baseline condition.

\section{Possible mechanisms of action}

We did not make any direct assessment of any possible mechanisms of action, although we had previously speculated about the role of social connectedness during the 12-month treatment phase ${ }^{13}$ and the possibility of a learning effect at the 24-month point of the study. ${ }^{11}$ One editorial speculated that the mechanism of action underpinning the reduction of self-harm reported by post-hospital contact interventions (such as Postcards from the EDge) might be because of the increased utilisation of mental health services as a result of the contact intervention. ${ }^{25}$ However, in our study the number of psychiatric admissions and number of in-patient days (i.e. access to in-patient care) were significantly less in the contact intervention group, suggesting this was not the mechanism of action. We did not have measures of other mental health service utilisation to further address this question. However, it is possible that increased use of mental health out-patient services, general practitioners or community-based counselling services might have occurred in the intervention group.

In HATS, the clinical toxicologist makes treatment decisions about the poisoning and fitness for discharge. In our study each recruiting toxicologist for any given participant was not masked to allocation. We cannot say that the treating toxicologists did not treat intervention and control groups differently in some way. However, many rather high-powered interventions have been tested and found to be unable to modify repeat rates of selfharm $^{26}$ even in the short term, so we doubt that any actions of the toxicologist would be able to produce the short-term and long-term beneficial effects demonstrated. We have very limited indirect evidence (Table 1) that the toxicologist used the same treatment for both groups; ICU admissions and length of stay in the general hospital were very similar.

Other authors have tried to identify what mechanisms might be useful in influencing repeat self-harm. A qualitative study of patients and service providers suggested that the period of time directly after discharge was the time of greatest need for contact and that patients would experience contact as a gesture of caring, which counteracted feelings of loneliness. ${ }^{27}$ The intervention should be both genuine in delivery and linked to current services. The authors suggested that an appropriate design for an intervention might be the provision of an information leaflet, telephone calls (soon after discharge), then letters (offering continuity of contact). ${ }^{27}$ The Postcards from the EDge intervention had some of these features. A review of attitudes to patients who self-harm in the general hospital suggested that poor communication with staff and a perceived lack of staff knowledge with regard to selfharm were common. Participants also suggested that psychosocial assessments and access to aftercare needed to be improved. ${ }^{28}$ The HATS model of clinical care, which underpins the Postcards from the EDge intervention, is based on coordinated multidisciplinary care, universal psychosocial assessment and referral to aftercare. ${ }^{14}$ The postcards intervention might not be effective at all if delivered within the context of an uncaring, uncoordinated general hospital service that did not provide comprehensive psychosocial assessment.

To examine potential mechanisms of treatment efficacy, future replication studies could use qualitative and quantitative methodologies to evaluate: patient experience of caring, level of loneliness, aspects of the therapeutic alliance including genuineness and promptness of contact, feelings of stigma, staff communication, referral to and subsequent use of out-patient services. Because of the possible differential response for females for the self-poisoning events outcome, it might be necessary to consider how these mechanisms of action might work in different genders.

Additionally, sustained beneficial effects (long after treatment cessation) on reduction in self-harm have been demonstrated in a limited number of studies for a variety of populations and interventions, which might involve different mechanisms of action to those mechanisms of action of benefit during the treatment period. 


\section{Benefit of treatment beyond the treatment phase}

Our study showed a reduction in long-term repetition of hospitaltreated self-poisoning events. A UK study of a brief nurse-led psychological intervention in patients who had self-poisoned, found fewer reports of repeated self-harm (9\% v. 28\%) 6 months after treatment, ${ }^{7}$ and a New Zealand study used a 3-month problem-solving intervention in patients who had self-harmed with a reduction in self-harm at 12 months only in the subgroup that had a history of self-harm at baseline $(13.5 \% v .22 .1 \%) .{ }^{29}$ In studies of individuals with borderline personality disorder, dialectical behaviour therapy reduced parasuicide 6 months after treatment ${ }^{30}$ and reduced self-harm 12 months after treatment $(23 \%$ v. $46 \%))^{10}$ and mentalisation-based treatment reduced self-mutilation $(23 \% v .68 \%)$ and suicidal gesture $(18 \% v .63 \%)$ 18 months after treatment ${ }^{8}$ and reduced 'suicidality' (23\% v. $74 \%) 5$ years after treatment. ${ }^{9}$ These studies used highly selected groups, individuals with borderline personality disorder, ${ }^{8,10}$ only 119 of 587 patients who self-poisoned ${ }^{7}$ or a subgroup with prior self-harm, ${ }^{29}$ which makes comparison with the current study difficult. Also, these studies examined formal psychological therapies, a very different approach to the sustained contact but not therapy model, used in the Postcards from the EDge project.

Nevertheless, these studies taken together suggest that if some impact on reduction of repetition of self-harm or self-poisoning behaviour can be made, the effects may be sustained over a period beyond the duration of the intervention. The mechanism of action for these sustained effects is unknown but suggests there must be some learning of alternate behaviours, which could be evaluated in future replication studies.

Our study also reported fewer psychiatric hospital admission events and psychiatric in-patient bed days. There are few comparable studies. In borderline personality disorder trials, for participants who received dialectical behaviour therapy, psychiatric hospital admissions were reduced after 12 months of treatment, ${ }^{31}$ while psychiatric in-patient days were no different for 12- to 18month follow-up, and reduced at 18 - to 24 -month follow-up; ${ }^{30}$ and for participants who received mentalisation-based treatment this reduced the proportion with any psychiatric admission, days of psychiatric hospital admission and out-patient contacts during 18 -month follow-up. ${ }^{8}$ The mentalisation group at 5-year follow-up also showed less psychiatric out-patient service use ( 2 v. 3.5 years of treatment). ${ }^{9}$ Taken together with our study, it may be that interventions that reduce self-harm might also reduce psychiatric service use during the period after treatment cessation.

\section{Comparison with other letter or postcard interventions}

The original Motto study used a letter writing intervention with psychiatric in-patients $(n=843)$ who refused all offers of clinical follow-up on discharge, reporting a reduction in suicide as the primary end-point in the first 2 years after hospital discharge. ${ }^{20}$ The Postcards from Persia study $(n=2300)$ showed a reduction in suicidal ideation and suicide attempt but no difference in self-cutting after 12 months of the intervention. ${ }^{24}$ The Postcards from the EDge project $(n=772)$ has previously reported a reduction in repetition of hospital-treated self-poisoning event rates but no difference in proportion of individual repeaters at 12 and 24 months, ${ }^{11,13}$ which has held true in the current study. The current study has also shown a reduction in event rates for psychiatric hospital admissions but no difference for proportion of individuals having any psychiatric admission. Taken together, these studies suggest that a brief, sustained postcard-type intervention might have some impact on a variety of suicidal behaviours and rates of hospital admissions, in different clinical populations.
The Christchurch postcards study was for individuals who self-harmed and were recruited from a psychiatric admission centre in New Zealand $(n=327)$. The results from this study are difficult to interpret but might be best seen as equivocal. The trial was powered to recruit 700 participants, but used a planned early stopping rule to cease recruitment at 327 participants because the event rates for further psychiatric emergency services visits was strongly in favour of the postcard intervention. This inadvertently resulted in the trial being substantially underpowered for the subsequent unplanned post hoc analyses. A later consideration of imbalances at baseline suggested an imbalance in favour of the intervention group for number of self-harm events in the 12 months before recruitment. The authors then undertook a post hoc analysis adjusting for the number of self-harm events before the study as a covariate and found that the significance level for this event-rate outcome (contact with psychiatric emergency services) was substantially reduced $(P=0.04)$.

\section{All-cause mortality and suicide deaths}

There were no differences in all-cause mortality and suicide deaths. The rate of all-cause mortality after 5 years was $5.7 \%$, which is very close to the rate of $5.6 \%$ for a mean follow-up of 5 years previously reported for HATS patients; ${ }^{32}$ and the suicide mortality after 5 years was $1.4 \%$ in the current study, exactly the same as previously reported for HATS patients. ${ }^{32}$

\section{Main findings}

In conclusion, this study showed that a low-cost postcard intervention continued to be effective over 5 years in reducing the number of events per individual for hospital-treated self-poisoning by around $50 \%$, which was clinically and statistically significant. This benefit was limited to females. The same intervention was also effective in reducing the number of events per individual for psychiatric hospital admissions by around 33\%, which was clinically and statistically significant overall but not significant within gender subgroups. For both primary outcomes, there was a benefit for patients with a baseline history of self-poisoning (although this was not statistically significant at the 5\% level for psychiatric hospital admissions), which was a subgroup we had not initially expected to respond to such a brief intervention.

The postcard intervention was effective in reducing repeat episodes of hospital-treated self-poisoning and psychiatric hospital admissions over the 5 years of the study. This represented an enormous opportunity for cost saving to the general hospital and psychiatric hospital systems (number of bed days available for other patients) for an intervention that was delivered to almost all individuals who self-poisoned (with few exclusions) presenting to a general hospital for treatment.

\footnotetext{
Gregory L. Carter, MBBS, FRANZCP, Cert Child Psych, PhD, Centre for Translational Neuroscience and Mental Health, Faculty of Health, University of Newcastle and Department of Consultation-Liaison Psychiatry, Calvary Mater Newcastle, Newcastle; Kerrie Clover, BSc (Hons), PhD, MAPS, Suicide Prevention Research Unit, Centre for Mental Health Studies and Faculty of Health, University of Newcastle, Newcastle; Ian M. Whyte, MBBS (Hons), FRACP, FRCP(Edin), FACMT, FAACT, Discipline of Clinical Pharmacology, Faculty of Health, University of Newcastle and Department of Clinical Toxicology and Pharmacology, Calvary Mater Newcastle, Newcastle; Andrew H. Dawson, MBBS, GradDip Epid, FRCP, FRACP, Sydney Medical School, University of Sydney and Department of Clinical Toxicology and Pharmacology, Calvary Mater Newcastle, Newcastle; Catherine D'Este, BMath, GradDip MedStat, DipEd, PhD, Centre for Clinical Epidemiology and Biostatistics, Faculty of Health, University of Newcastle and Centre for Military and Veterans' Health, University of Queensland, Hurston, Australia
}

Correspondence: Gregory Carter, Department of Consultation-Liaison Psychiatry, Locked Bag \#7, Hunter Region Mail Centre NSW 2310, Australia. Email: gregory.carter@newcastle.edu.au

First received 29 Mar 2012, final revision 6 Nov 2012, accepted 21 Jan 2013 


\section{Funding}

NSW Health, Burdekin Mental Health Enhancement Strategy for K.C.'s position.

\section{Acknowledgements}

We thank Dr Jerome Motto for his encouragement to use and modify the original intervention for this project. We also acknowledge the contribution of Drs Trish McGettigan, Patrick Oakley, Nav Gupta and Geoff Isbister for patient recruitment and Mr Stuart Allen for data management support. We would also like to thank Professor Bob Goldney and Dr Natalia Carter for alerting us to the Motto study, Deb Whyte and Antonia Nash for data entry, Dr Julia Lowe and Dr Dianne O'Connell for schooling us in Zelen designs, Andrew Landers and lan Schumacher for the extraction of psychiatric hospital data from the HNEAHS information system and John Harding for the AlHW mortality data. Finally, we thank Mrs Helen Rowsell, the departmental secretary, who managed the mailing database and mailing lists so diligently over a long period.

\section{Reference}

1 McGrath J. A survey of deliberate self-poisoning. Med J Aust 1989; 150: 317-22.

2 Berry JG, Harrison JE. Hospital Separations due to Injury and Poisoning, Australia 2003-04. Cat. no. INJCAT 88. AlHW, 2007

3 House A, Owens D, Patchett L. Deliberate self-harm. Eff Health Care 1998; 4: 1-12.

4 Lilley R, Owens D, Horrocks J, House A, Noble R, Bergen H, et al. Hospital care and repetition following self-harm: multicentre comparison of selfpoisoning and self-injury. Br J Psychiatry 2008; 192: 440-5.

5 Gunnell D, Bennewith O, Peters TJ, House A, Hawton K. The epidemiology and management of self-harm amongst adults in England. $J$ Public Health (Oxf) 2005; 27: 67-73.

6 Owens D, Horrocks J, House A. Fatal and non-fatal repetition of self-harm. Systematic review. Br J Psychiatry 2002; 181: 193-9.

7 Guthrie E, Kapur N, Mackway-Jones K, Chew-Graham C, Moorey J, Mendel E, et al. Randomised controlled trial of brief psychological intervention after deliberate self poisoning. BMJ 2001; 323: 135-8.

8 Bateman A, Fonagy P. Treatment of borderline personality disorder with psychoanalytically oriented partial hospitalization: an 18-month follow-up. Am J Psychiatry 2001; 158: 36-42.

9 Bateman A, Fonagy P. 8-year follow-up of patients treated for borderline personality disorder: mentalization-based treatment versus treatment as usual. Am J Psychiatry 2008; 165: 631-8.

10 Linehan MM, Comtois KA, Murray AM, Brown MZ, Gallop RJ, Heard HL, et al. Two-year randomized controlled trial and follow-up of dialectical behavior therapy vs therapy by experts for suicidal behaviors and borderline personality disorder. Arch Gen Psychiatry 2006; 63: 757-66.

11 Carter GL, Clover K, Whyte IM, Dawson AH, D'Este C. Postcards from the EDge: 24-month outcomes of a randomised controlled trial for hospital-treated self-poisoning. Br J Psychiatry 2007; 191: 548-53.

12 Bateman A, Fonagy P. Health service utilization costs for borderline personality disorder patients treated with psychoanalytically oriented partial hospitalization versus general psychiatric care. Am J Psychiatry 2003; 160: 169-71.

13 Carter GL, Clover K, Whyte IM, Dawson AH, D'Este C. Postcards from the EDge project: randomised controlled trial of an intervention using postcards to reduce repetition of hospital treated deliberate self poisoning. BMJ 2005; 331: 805-7.

14 Whyte IM, Dawson AH, Buckley NA, Carter GL, Levey CM. A model for the management of self-poisoning. Med J Aust 1997; 167: 142-6.

15 Whyte IM, Buckley NA, Dawson AH. Data collection in clinical toxicology: are there too many variables? J Toxicol Clin Toxicol 2002; 40: 223-30.

16 Buckley NA, Whyte IM, Dawson AH, Reith D. Preformatted admission charts for poisoning admissions facilitate clinical assessment and research. Ann Emerg Med 1999; 34: 476-82.

17 Zelen M. A new design for randomized clinical trials. N Engl J Med 1979; 300 1242-5.

18 Zelen M. Randomized consent designs for clinical trials: an update. Stat Med 1990; 9: 645-56.

19 Motto JA. Suicide prevention for high-risk persons who refuse treatment. Suicide Life Threat Behav 1976; 6: 223-30.

20 Motto JA, Bostrom AG. A randomized controlled trial of postcrisis suicide prevention. Psychiatr Serv 2001; 52: 828-33.

21 Carter GL, Whyte IM, Ball K, Carter NT, Dawson AH, Carr VJ, et al. Repetition of deliberate self-poisoning in an Australian hospital-treated population. Med J Aust 1999; 170: 307-11.

22 Carter GL, Clover KA, Bryant JL, Whyte IM. Can the Edinburgh Risk of Repetition Scale predict repetition of deliberate self-poisoning in an Australian clinical setting? Suicide Life Threat Behav 2002; 32: 230-9.

23 Carter GL, Safranko I, Lewin TJ, Whyte IM, Bryant JL. Psychiatric hospitalization after deliberate self-poisoning. Suicide Life Threat Behav 2006; 36: $213-22$.

24 Hassanian-Moghaddam H, Sarjami S, Kolahi A-A, Carter GL. Postcards in Persia: randomised controlled trial to reduce suicidal behaviours 12 months after hospital-treated self-poisoning. Br J Psychiatry 2011; 198: 309-16.

25 Kapur N, Cooper J, Bennewith O, Gunnell D, Hawton K. Postcards, green cards and telephone calls: therapeutic contact with individuals following selfharm. Br J Psychiatry 2010; 197: 5-7.

26 Hawton KE, Townsend E, Arensman E, Gunnell D, Hazell P, House A, et al. Psychosocial and pharmacological treatments for deliberate self harm. Cochrane Database Syst Rev 1999; 4: CD001764.

27 Cooper J, Hunter C, Owen-Smith A, Gunnell D, Donovan J, Hawton K, et al. "Well it's like someone at the other end cares about you." A qualitative study exploring the views of users and providers of care of contact-based interventions following self-harm. Gen Hosp Psychiatry 2011; 33: 166-76.

28 Taylor TL, Hawton K, Fortune S, Kapur N. Attitudes towards clinical services among people who self-harm: systematic review. Br J Psychiatry 2009; 194: 104-10.

29 Hatcher S, Sharon C, Parag V, Collins N. Problem-solving therapy for people who present to hospital with self-harm: Zelen randomised controlled trial. Br J Psychiatry 2011; 199: 310-6.

30 Linehan MM, Heard HL, Armstrong HE. Naturalistic follow-up of a behavioral treatment for chronically parasuicidal borderline patients. Arch Gen Psychiatry 1993; 50: 971-4.

31 Linehan MM, Armstrong HE, Suarez A, Allmon D, Heard HL. Cognitivebehavioral treatment of chronically parasuicidal borderline patients. Arch Gen Psychiatry 1991; 48: 1060-4.

32 Reith DM, Whyte IM, Carter GL, McPherson M, Carter NT. Risk factors for suicide and other deaths following hospital treated self-poisoning in Australia. Aust N Z J Psychiatry 2004; 38: 520-5. 